\title{
Elaboração e caracterização de geleia prebiótica mista de jabuticaba e acerola
}

\section{Elaboration and characterization of jabuticaba and acerola prebiotic jelly}

\author{
Danielle Martins Lemos ${ }^{1 *}$ (1), Ana Paula Trindade Rocha², \\ Josivanda Palmeira Gomes de Gouveia², Emanuel Neto Alves de Oliveira ${ }^{3}$, \\ Elisabete Piancó de Sousa ${ }^{4}$, Shirlyanne Ferreira da Silva ${ }^{5}$
}

${ }^{1}$ Instituto Federal de Alagoas (IFAL), Curso Técnico em Agroindústria, Batalha/AL - Brasil

${ }^{2}$ Universidade Federal de Campina Grande (UFCG), Centro de Tecnologia e Recursos Naturais, Campina

Grande/PB - Brasil

${ }^{3}$ Instituto Federal do Rio Grande do Norte (IFRN), Ciência e Tecnologia de Alimentos, Pau dos Ferros/RN - Brasil

${ }^{4}$ Instituto Federal do Amapá (IFAP), Curso Tecnólogo em Alimentos, Macapá/AM - Brasil

${ }^{5}$ Universidade Federal de Campina Grande (UFCG), Unidade Acadêmica de Engenharia Agrícola, Engenharia

Agrícola, Campina Grande/PB - Brasil

${ }^{*}$ Corresponding Author: Danielle Martins Lemos, Instituto Federal de Alagoas (IFAL), Curso Técnico em Agroindústria, Av. Afrânio Lages, 391-453, CEP: 57420-000, Batalha/AL - Brasil, e-mail:

danielemartinsali@gmail.com

Cite as: Lemos, D. M., Rocha, A. P. T., Gouveia, J. P. G., Oliveira, E. N. A., Sousa, E. P., \& Silva, S. F. (2019).

Elaboration and characterization of jabuticaba and acerola prebiotic jelly. Brazilian Journal of Food Technology, 22, e2018098. https://doi.org/10.1590/1981-6723.09818

\begin{abstract}
Resumo
Objetivou-se, com este trabalho, a elaboração e a caracterização físico-química de geleias tradicionais prebióticas mistas de jabuticaba e acerola. As formulações compostas de polpas e blends foram: $F_{1}$ ( $75 \%$ acerola/25\% jabuticaba), $F_{2}\left(50 \%\right.$ acerola/50\% jabuticaba), $F_{3}$ (25\% acerola/75\% jabuticaba), $F_{4}\left(100 \%\right.$ jabuticaba) e $F_{5}(100 \%$ acerola) com $1,0 \%$ de pectina, $0,1 \%$ de benzoato de potássio, $3 \%$ de inulina e $40 \%$ de sacarose. Os ingredientes foram misturados e as misturas foram concentradas até atingir sólidos solúveis totais (SST) entre 65 e $68^{\circ}$ Brix. As geleias a partir de polpas e blends de acerola e jabuticaba indicaram teores de acidez com variação de $0,77 \%$ a $0,80 \%$. As geleias $F_{1}$ e $F_{5}$, as quais possuem a maior concentração de polpa de acerola na formulação, apresentaram SST de $65^{\circ}$ Brix. Verificou-se que na proporção em que se aumentou a porcentagem de polpa de jabuticaba nas geleias, diminuiu-se o teor de polifenóis totais de 405,43 para 135,75 GAEg/100 g. Observou-se que à medida que se aumentou a proporção de polpa de acerola nos blends, aumentou-se também os valores de vitamina $C$, com destaque na polpa de acerola pura com $3704,50 \mathrm{mg} / 100 \mathrm{~g}$. Quanto aos carotenoides totais, verificou-se variação de 1,63 a 4,62 mg/100 g. A geleia $\mathrm{F}_{4}$, obtida a partir de polpa e casca de jabuticaba, apresentou os maiores valores para os parâmetros de textura, exceto para coesividade. Observou-se que os teores de compostos antioxidantes preconizam um perfil nutricional às geleias. Quanto ao perfil funcional, o produto atende à legislação brasileira em vigor por conter inulina, além da presença de antioxidantes.
\end{abstract}

Palavras-chave: Plinia cauliflora; Malpighia punicifolia L.; Blends de frutas; Compostos bioativos; Alimento funcional; Perfil de textura. 


\begin{abstract}
The objective of this work was the elaboration and physicochemical characterization of traditional mixed prebiotic jellies of jabuticaba and acerola. In the case of $\mathrm{F} 2$ ( $50 \%$ acerola/50\% jabuticaba), F3 ( $25 \%$ acerola/75\% jabuticaba), F4 (100\% jabuticaba) and F5 (100\% acerola) with $1.0 \%$ pectin, $0.1 \%$ potassium benzoate, $3 \%$ inulin and $40 \%$ sucrose. The ingredients were mixed and the mixtures were concentrated to total soluble solids (TSS) between 65 and $68^{\circ}$ Brix. The jellies from pulps and blends of acerola and jabuticaba presented acidity levels varying from $0.77 \%$ to $0.80 \%$. The jellies F1 and F5, which have the highest concentration of acerola pulp in the formulation, presented TSS of $65^{\circ}$ Brix. It has been found that as the percentage of jaboticaba pulp increased in the jellies, the total polyphenol content decreased from 405.43 to $135.75 \mathrm{GAEg} / 100 \mathrm{~g}$. It is observed that, as the proportion of acerola pulp in the blends increased, the vitamin C content also increased, especially in pure acerola pulp with $3,704.50 \mathrm{mg} / 100 \mathrm{~g}$. Total carotenoids ranged from 1.63 to $4.62 \mathrm{mg} / 100 \mathrm{~g}$. F4 jelly, obtained from jabuticaba pulp and peel, presented the highest values for texture parameters, except for cohesiveness. It was observed that the contents of antioxidant compounds favored a nutritional profile to the jellies. Regarding the functional profile, the product complies with the current Brazilian legislation because it contains inulin, in addition to the presence of antioxidants.
\end{abstract}

Keywords: Plinia cauliflora; Malpighia punicifolia L.; Fruit blends; Bioactive compounds; Functional food; Texture profile.

\title{
1 Introdução
}

A jabuticaba (Plinia cauliflora) é uma fruta da família Myrtaceae, amplamente distribuída no território brasileiro, e apresenta-se sob a forma de uma baga globosa, com até $3 \mathrm{~cm}$ de diâmetro, casca avermelhada quase preta, polpa esbranquiçada, mucilaginosa, agridoce, saborosa, comumente com uma única semente (Lima et al., 2008). Algumas formas de aproveitamento da fruta podem ser verificadas na fabricação de geleias, vinhos, sucos, além do consumo in natura (Barbosa et al., 2017).

A acerola (Malpighia punicifolia L.) tem como característica polpa suculenta e casca protetora que, ao amadurecer, muda a coloração de verde para amarelo-avermelhado e finalmente para vermelho ou roxo, quando completamente amadurecida, dependendo da variedade. A mudança de pigmento está ligada a mudanças bioquímicas complexas que envolvem todos os seus principais compostos, como carotenos, tiamina, riboflavina, niacina, cálcio, fósforo e vitamina C (Malegori et al., 2017).

Por possuírem características peculiares, as polpas de jabuticaba e acerola podem ser utilizadas na elaboração de blends, os quais consistem na mistura de um ou mais tipos de polpas, conferindo novas características sensoriais e nutricionais ao produto. Os blends de frutas podem ser utilizados para o processamento de vários produtos com a finalidade de agradar o paladar dos consumidores, entre os quais a elaboração de geleias.

Algumas pesquisas têm sido realizadas quanto ao desenvolvimento de geleias a partir de blends de frutas, as quais proporcionam o aumento ou o equilíbrio entre nutrientes presentes na matéria-prima, seja vitamina C ou os demais compostos bioativos, Logo, foram estudadas a produção de geleias mistas de acerola e manga (Maciel et al., 2009); melancia e tamarindo (Ferreira et al., 2011); de araçá e banana (Viana et al., 2014); laranja e acerola (Pelegrine et al., 2015), e pêssego e abacaxi (Vieira et al., 2017).

De acordo com a Resolução n. ${ }^{\circ}$ 272, de 22 de setembro de 2005 (Brasil, 2005), as geleias são os produtos elaborados a partir de fruta(s), inteira(s) ou em parte(s), e/ou semente(s), obtidos por cocção e/ou concentração, e/ou outros processos tecnológicos considerados seguros para a produção de alimentos, podendo ser apresentados com ou sem líquido de cobertura e adicionados de açúcar, sal, tempero, especiaria e/ou outro ingrediente, desde que não descaracterize o produto. Entretanto, outros ingredientes podem ser acrescentados ao produto, como prebióticos, os quais são componentes alimentares não digeríveis que afetam beneficamente a saúde, por incitarem seletivamente a proliferação ou a atividade de populações de bactérias 
desejáveis no cólon e inibir a multiplicação de patógenos (Uyeda et al., 2017). Dentre as substâncias prebióticas, destaca-se a inulina, a qual tem numerosas características benéficas como ingrediente funcional, oferecendo uma combinação única de propriedades nutricionais distintas e benefícios tecnológicos importantes, podendo melhorar o sabor e a textura em muitos alimentos (Chaito et al., 2014). Pesquisas são realizadas quanto à adição de inulina na elaboração de produtos prebióticos: Munhoz et al. (2018) produziram iogurte com geleia de jambo vermelho com $0,7 \%$; bebidas mistas com propriedades funcionais, à base de polpa de graviola e água de coco, foram elaboradas por Oliveira et al. (2017) com 2\%; Monteiro et al. (2015) elaboraram um sorvete de iogurte, à base de leite de búfala, com propriedade simbiótica e saborizado com açaí com $1 \%$.

Diante da escassez de pesquisas referenciadas à utilização de blends de jabuticaba e acerola, objetivou-se, com este trabalho, a elaboração e a caracterização físico-química e de compostos bioativos de geleias prebióticas tradicionais mistas de jabuticaba e acerola.

\section{Material e métodos}

\subsection{Obtenção e processamento dos frutos}

Os frutos foram obtidos na Central Estadual de Abastecimento (CEASA) na cidade de Campina GrandePB e transportados para o Laboratório de Engenharia de Alimentos (LEA) da Universidade Federal de Campina Grande (UFCG).

Foram selecionados os frutos sadios, lavados em água corrente e depois sanitizados por imersão em uma solução de hipoclorito de sódio a $50 \mathrm{ppm}$, durante 10 minutos, sendo, posteriormente, enxaguados em água corrente. Os frutos de jabuticaba e acerola foram despolpados em despolpadeira horizontal da marca Laboremus, modelo DF-200, utilizando-se peneira de malha 2,5 mm.

A polpa de jabuticaba foi adicionada de $10 \%$ de cascas de jabuticaba (quantidade definida por meio de pré-testes), que foram trituradas e homogeneizadas em liquidificador. As polpas obtidas foram acondicionadas em embalagens de polietileno com capacidade para $500 \mathrm{~g}$ e armazenadas a $-18^{\circ} \mathrm{C}$ em freezer horizontal da marca Consul com capacidade de 519 L, modelo CHB53EB.

\subsection{Preparação dos blends e processamento das geleias}

A elaboração dos blends das polpas deu-se da seguinte forma: as polpas foram descongeladas sob refrigeração a $4 \pm 2{ }^{\circ} \mathrm{C}$, pesadas e misturadas. As formulações foram: $\mathrm{F}_{1}\left(75 \%\right.$ acerola/25\% jabuticaba), $\mathrm{F}_{2}$ ( $50 \%$ acerola $/ 50 \%$ jabuticaba), $\mathrm{F}_{3}\left(25 \%\right.$ acerola $/ 75 \%$ jabuticaba), $\mathrm{F}_{4}\left(100 \%\right.$ jabuticaba) e $\mathrm{F}_{5}$ ( $100 \%$ acerola). As polpas de jabuticaba e acerola, e os blends tiveram pH corrigido para 3,2 com bicarbonato de sódio de grau alimentício.

As geleias tradicionais foram elaboradas tendo como base $4 \mathrm{~kg}$ de polpa no total para cada formulação e elaboradas como tipo Premium (40\% açúcar/60\% polpa), de acordo com Furlaneto et al. (2015). Os ingredientes acrescentados nas formulações tiveram quantidades referentes ao total de açúcar, os quais foram: benzoato de potássio $(0,1 \%)$ e pectina de Alto Teor de Metoxilação - ATM $(1,0 \%)$ com grau de $150^{\circ}$ US SAG e velocidade de gelificação rápida, cedida pela $\mathrm{CP}$ Kelco Brasil.

A polpa foi aquecida até aproximadamente $70{ }^{\circ} \mathrm{C}$ e logo foi adicionado $70 \%$ do açúcar. Próximo ao ponto final de cozimento, com temperatura acima de $100^{\circ} \mathrm{C}$, foram adicionadas a pectina $(1,0 \%)$ e a inulina $(3,0 \%)$, misturadas ao restante de açúcar.

As formulações foram concentradas em um tacho encamisado inoxidável com agitação mecânica até teor de sólidos solúveis totais com variação de 65 a $68{ }^{\circ}$ Brix. Em razão de serem cinco formulações, não foi possível a padronização do ${ }^{\circ}$ Brix final de forma homogênea em todas. Posteriormente à etapa de 
concentração, as geleias foram acondicionadas em recipientes de vidro com capacidade de $600 \mathrm{~mL}$ com tampas metálicas, resfriadas por imersão em água fria até atingir temperatura de $\pm 25^{\circ} \mathrm{C}$ e colocadas ao abrigo da luz e à temperatura ambiente $\left( \pm 25^{\circ} \mathrm{C}\right)$ por 8 dias, até serem submetidas às análises físicas e físicoquímicas.

\subsection{Análises físicas, físico-químicas e de compostos bioativos}

As análises físicas e físico-químicas das polpas, dos blends e das geleias foram realizadas em triplicata, de acordo com o Instituto Adolfo Lutz (2008): pH; sólidos solúveis totais (SST); teor de água; cinzas; acidez total titulável (ATT em porcentagem de ácido cítrico), e vitamina C. Os açúcares totais em glicose, redutores em glicose e não redutores em sacarose foram determinados pelo método de Lane e Eynon, descrito por Ranganna (1986). A atividade de água ( $\mathrm{a}_{\mathrm{w}}$ ) foi medida em um instrumento digital Aqua-Lab 3TE fabricado pela Decagon Devices Inc., EUA.

As características de cor foram avaliadas através de um espectrofotômetro portátil Hunter Lab Mini Scan XE Plus, modelo $4500 \mathrm{~L}$, com obtenção dos parâmetros luminosidade $\left(\mathrm{L}^{*}\right)$, intensidade de vermelho $\left(+\mathrm{a}^{*}\right)$ e intensidade de amarelo $\left(+b^{*}\right)$. A Análise do Perfil de Textura (TPA) foi determinada conforme metodologia de Dias et al. (2011), com resultados obtidos da curva, força $\times$ tempo, calculados pelo Software Texture Expert Versão 1.22, com avaliação dos parâmetros de firmeza, adesividade, coesividade, elasticidade, gomosidade e mastigabilidade.

Quanto à determinação de compostos bioativos, foram determinadas antocianinas (Francis, 1982), carotenoides (Ramalho, 2005) e o índice de polifenóis totais (Singleton \& Rossi, 1965).

Para análise estatística dos dados, foi usado delineamento inteiramente casualizado (DIC) no software ASSISTAT versão 7.7 Beta (Silva \& Azevedo, 2016), com uso da comparação entre médias por meio do teste de Tukey.

\section{Resultados e discussões}

\subsection{Caracterização física e físico-química das polpas e blends}

Verificou-se que as amostras $\mathrm{F}_{1}, \mathrm{~F}_{2}$ e $\mathrm{F}_{3}$, compostas pelos blends, apresentaram teor de água (Tabela 1) intermediário entre a polpa de jabuticaba $(90,29 \%)$, que apresentou o menor valor, e a polpa de acerola, o maior $(94,64 \%)$, sendo que a casca adicionada à polpa de jabuticaba influenciou diretamente esses resultados. Valor próximo (92,0\%) ao encontrado para a polpa de acerola é reportado por Mercali et al. (2011).

Dessimoni-Pinto et al. (2011), ao estudarem o aproveitamento tecnológico da jabuticaba, identificaram para casca e polpa valores de cinzas de $0,54 \%$ e $0,06 \%$, respectivamente. De acordo com Universidade Estadual de Campinas (2011), a polpa de acerola equivale a 0,4\% de teor de cinzas. Entretanto, observam-se valores menores para a polpa de acerola $(0,22 \%)$ e seus respectivos blends, com $0,21 \%, 0,22 \%$ e $0,28 \%$, enquanto valor de $0,25 \%$ foi identificado na polpa de jabuticaba com casca. De acordo com Uchoa et al. (2008), maiores taxas de cinzas podem estar acompanhadas de uma alta concentração dos minerais. Tal fato demonstra que a polpa de jabuticaba pode possuir maior concentração de minerais do que a polpa de acerola.

Com relação à atividade de água $\left(\mathrm{A}_{\mathrm{w}}\right)$, os valores obtidos variaram de 0,988 a 0,993 , e não diferiram estatisticamente entre si para $F_{1}, F_{2}$ e $F_{4}$. Em polpa de acerola analisada por Nóbrega et al. (2015), foi encontrado 0,989 de $\mathrm{a}_{\mathrm{w}}$. Com valores superiores a 0,99 nas matérias-primas, averíguou-se a precisão de processamento higiênico-sanitário rigoroso e armazenamento em temperatura de congelação. 
Tabela 1. Valores médios das análises físico-químicas dos blends e polpas.

\begin{tabular}{|c|c|c|c|c|c|c|c|}
\hline \multirow{2}{*}{ Parâmetros } & \multicolumn{7}{|c|}{ Blends e polpas } \\
\hline & $\mathrm{F}_{1}$ & $\mathrm{~F}_{2}$ & $\mathrm{~F}_{3}$ & $\mathrm{~F}_{4}$ & $\mathrm{~F}_{5}$ & $\mathrm{CV} \%$ & $\mathrm{MG}$ \\
\hline Teor de água (\%) & $93,75^{\mathrm{a}}$ & $90,94^{\mathrm{b}}$ & $91,28^{b}$ & $90,29^{b}$ & $94,64^{\mathrm{a}}$ & 0,83 & 92,18 \\
\hline Cinzas $(\%)$ & $0,21^{\mathrm{c}}$ & $0,22^{\mathrm{c}}$ & $0,28^{a}$ & $0,25^{\mathrm{b}}$ & $0,22^{\mathrm{c}}$ & 2,69 & 0,23 \\
\hline $\mathrm{A}_{\mathrm{W}}$ & $0,992^{\mathrm{a}}$ & $0,993^{\mathrm{a}}$ & $0,988^{b}$ & $0,991^{\mathrm{a}}$ & $0,988^{b}$ & 0,10 & 0,99 \\
\hline ATT $(\%)$ & $1,09^{c}$ & $1,22^{b}$ & $1,24^{\mathrm{b}}$ & $1,32^{\mathrm{a}}$ & $1,14^{\mathrm{c}}$ & 2,36 & 1,20 \\
\hline $\mathrm{pH}$ & $2,97^{\mathrm{b}}$ & $2,87^{\mathrm{c}}$ & $2,90^{\mathrm{c}}$ & $2,88^{\mathrm{c}}$ & $3,03^{\mathrm{a}}$ & 0,52 & 2,93 \\
\hline SST $\left({ }^{\circ}\right.$ Brix $)$ & $6,87^{\mathrm{d}}$ & $8,63^{\mathrm{c}}$ & $8,83^{\mathrm{b}}$ & $9,63^{\mathrm{a}}$ & $5,06^{\mathrm{e}}$ & 0,94 & 7,80 \\
\hline $\mathrm{AR}(\%)$ & $9,94^{\mathrm{c}}$ & $11,09^{b}$ & $14,35^{\mathrm{a}}$ & $11,07^{\mathrm{b}}$ & $7,17^{\mathrm{d}}$ & 0,87 & 10,72 \\
\hline AT $(\%)$ & $8,34^{\mathrm{c}}$ & $9,42^{\mathrm{b}}$ & $10,76^{\mathrm{a}}$ & $7,52^{\mathrm{d}}$ & $5,78^{\mathrm{e}}$ & 0,22 & 8,36 \\
\hline ANR $(\%)$ & $2,45^{\mathrm{b}}$ & $2,83^{\mathrm{a}}$ & $2,30^{\mathrm{c}}$ & $1,04^{\mathrm{e}}$ & $1,54^{\mathrm{d}}$ & 2,65 & 2,03 \\
\hline Vitamina C (mg/100 g) & $1674,35^{\mathrm{b}}$ & $853,95^{\mathrm{c}}$ & $482,25^{\mathrm{d}}$ & $10,80^{\mathrm{e}}$ & $3704,50^{\mathrm{a}}$ & 6,27 & 1345,17 \\
\hline Luminosidade $\left(\mathrm{L}^{*}\right)$ & $33,33^{\mathrm{c}}$ & $36,06^{\mathrm{b}}$ & $28,51^{\mathrm{d}}$ & $24,23^{\mathrm{e}}$ & $41,06^{\mathrm{a}}$ & 0,92 & 32,63 \\
\hline$+a^{*}$ & $19,61^{b}$ & $18,61^{\mathrm{c}}$ & $16,28^{\mathrm{d}}$ & $15,64^{\mathrm{e}}$ & $27,69^{\mathrm{a}}$ & 0,79 & 15,57 \\
\hline$+b^{*}$ & $31,00^{\mathrm{b}}$ & $25,23^{\mathrm{c}}$ & $17,19^{\mathrm{d}}$ & $17,25^{\mathrm{d}}$ & $41,36^{\mathrm{a}}$ & 2,02 & 26,4 \\
\hline Antocianinas $(\mathrm{mg} / 100 \mathrm{~g})$ & $6,11^{\mathrm{b}}$ & $5,39^{c}$ & $4,50^{\mathrm{d}}$ & $2,92^{\mathrm{e}}$ & $6,79^{a}$ & 0,18 & 5,13 \\
\hline $\mathrm{CT}(\mu \mathrm{g} / \mathrm{g})$ & $556,15^{\mathrm{a}}$ & $902,39^{\mathrm{b}}$ & $750,00^{c}$ & $717,97^{\mathrm{c}}$ & $530,72^{\mathrm{d}}$ & 3,48 & 891,44 \\
\hline PT (GAEg/100 g) & $240,50^{b}$ & $201,64^{c}$ & $116,27^{d}$ & $51,00^{\mathrm{e}}$ & $256,22^{\mathrm{a}}$ & 0,20 & 173,12 \\
\hline
\end{tabular}

$\mathrm{F}_{1}=75 \%$ acerola $/ 25 \%$ jabuticaba. $\mathrm{F}_{2}=50 \%$ acerola $/ 50 \%$ jabuticaba. $\mathrm{F}_{3}=25 \%$ acerola $/ 75 \%$ jabuticaba. $\mathrm{F}_{4}=100 \%$ jabuticaba. $\mathrm{F}_{5}=100 \%$ acerola. $\mathrm{A}_{\mathrm{w}}=$ atividade de água. ATT = acidez total titulável. $\mathrm{SST}=$ sólidos solúveis totais. $\mathrm{AR}=$ açúcar redutor. AT = açúcar total. $\mathrm{ANR}=$ açúcar não-redutor. $+\mathrm{a}^{*}=$ intensidade de vermelho. $+\mathrm{b}^{*}=$ intensidade de amarelo. $\mathrm{CT}=$ carotenoides totais. $\mathrm{PT}=$ polifenóis totais. $\mathrm{CV}(\%)=$ coeficiente de variação. $\mathrm{MG}=$ média geral. Obs.: Médias seguidas da mesma letra na linha não diferem estaticamente pelo teste de Tukey, a $5 \%$ de probabilidade.

As Formulações $F_{2}$ e $F_{3}$ não diferiram estatisticamente entre si quanto aos parâmetros $\mathrm{pH}$ e acidez. Reis et al. (2017), ao avaliarem o pH de acerola, obtiveram valor de 3,47, que se mostra superior às formulações de blends e polpas puras analisadas neste trabalho. $\mathrm{O}$ teor de acidez da polpa de jabuticaba $(1,32 \%)$ indicou valor superior ao da polpa de acerola $(1,14 \%)$. Provavelmente, essa diferença ocorreu porque a acerola está no estágio de maturação mais avançado que o da jabuticaba, pois, nesse estádio, sabores e odores específicos desenvolvem-se em conjunto com o aumento da doçura, com a redução da acidez e da adstringência (Chitarra \& Chitarra, 2005). Segundo Lima et al. (2011), a polpa e a casca da jabuticaba apresentam pH ácido, elevada quantidade de ácidos orgânicos, ácido cítrico, ácido succínico, ácido málico, ácido oxálico e ácido acético, em ordem decrescente quantitativa de ácido. Deve-se considerar também que, no beneficiamento, quando as frutas passam pela despolpadeira, grande parte dos ácidos fica retida nas sementes e na película.

Quanto aos SST, observou-se que à medida que se aumentou a quantidade de polpa de jabuticaba nas formulações, o valor do ${ }^{\circ}$ Brix aumentou $\left(6,87,8,63\right.$ e $8,83{ }^{\circ}$ Brix para $F_{1}, F_{2}$ e $F_{3}$, respectivamente). Em polpa de jabuticaba analisada por Becker et al. (2015), foi verificado valor de $8,0^{\circ}$ Brix, inferior ao da polpa de jabuticaba do presente estudo. Frutas com maiores valores de SST favorecem a adição de menor quantidade de açúcar ao produto para atingir a concentração final (Lamounier et al., 2015).

Os valores de açúcares redutores e açúcares totais da polpa de acerola foram 7,17\% e 5,78\%, respectivamente; já os encontrados por Caetano et al. (2012) foram respectivamente inferiores, 5,26\% e $5,14 \%$, e, provavelmente, esta diferença pode ser atribuída ao estado de maturação da fruta. Salamoni et al. (2015), ao avaliarem a casca e a polpa de jabuticaba, indicaram teores de $8,16 \%$ e 4,86\% de açúcares totais, respectivamente. Tal discrepância observada na polpa de jabuticaba em estudo $(7,52 \%)$ deveu-se à mistura de casca e polpa de jabuticaba.

Os teores de vitamina $\mathrm{C}$ diferiram estatisticamente entre si para as amostras em geral (Tabela 1). Observouse, para ácido ascórbico, que à medida que se aumentou a proporção de polpa de acerola nos blends, aumentou-se também os valores de vitamina C. Na polpa de acerola pura, o valor encontrado $(3.704,50 \mathrm{mg} / 100 \mathrm{~g})$ foi superior ao analisado por Figueiredo Neto et al. (2017), de $3.260 \mathrm{mg} / 100 \mathrm{~g}$. 
A amostra $\mathrm{F}_{4}\left(100 \%\right.$ jabuticaba) apresentou luminosidade $\left(\mathrm{L}^{*}\right)$ de 24,23 , convergindo para uma amostra de tonalidade escura; com valores de intensidade de vermelho ( $\left.+\mathrm{a}^{*}\right)$ de 15,64 e de amarelo $\left(+\mathrm{b}^{*}\right)$ de 17,25, apresentou assim maiores tendências para o amarelo do que para o vermelho. $\mathrm{O}$ mesmo foi verificado por Nunes et al. (2014) para polpa de jabuticaba congelada, em que se observaram valores de $L^{*}$ de $21,57,+a^{*}$ de 11,40 e de $+b^{*}$ de 13,99 .

O teor de antocianinas (Tabela 1) da polpa de acerola $(6,79 \mathrm{mg} / 100 \mathrm{~g})$ corroborou com os valores encontrados por Rosso \& Mercadante (2007) de 7,21 mg/100 g em acerola in natura. Verificou-se que à medida que se reduziu a porcentagem de acerola nos blends diminuiu também o valor de antocianinas. Quanto à polpa de jabuticaba, notou-se valor de $2,92 \mathrm{mg} / 100 \mathrm{~g}$, enquanto Rufino et al. (2011) identificaram $58,1 \mathrm{mg} / 100 \mathrm{~g}$ de antocianinas totais. A discrepância entre os resultados deveu-se à exposição do congelamento lento no momento da armazenagem, pois a deficiência natural de elétrons das antocianinas, os quais são particularmente reativos, apresentaram também uma grande sensibilidade às mudanças de $\mathrm{pH} \mathrm{e}$ temperatura (Volp et al., 2008).

Na acerola, a coloração amarela é conferida pelos carotenoides e essa afirmação pode ser comprovada no estudo em questão, pois a polpa de acerola apresentou índice de carotenoides maiores $\left(717,97 \mu \mathrm{g} \mathrm{g}^{-1}\right)$, juntamente com seus blends $\left(556,15,902,39\right.$ e 750,00 $\left.\mu \mathrm{g} \mathrm{g}^{-1}\right)$. Assim, é possível afirmar que a utilização de blends de frutas com a acerola para a produção de novos produtos é de grande valia, pois os mesmos terão em sua composição característica de potencial antioxidante benéfico à saúde.

Dentre as polpas e blends analisados, a polpa de acerola apresentou o menor valor para carotenoides totais, $530,72 \mu \mathrm{g} \mathrm{g}^{-1}$. Aquino et al. (2011), ao armazenarem o fruto da acerola sob congelamento, observaram teor de 332,11 $\mu \mathrm{g} \mathrm{g}^{-1}$. Apesar da coloração arroxeada, observou-se um alto teor de carotenoides na polpa de jabuticaba adicionada de cascas $\left(717,97 \mu \mathrm{g} \mathrm{g}^{-1}\right)$, pois, em produtos vegetais, os carotenoides encontram-se associados nos cloroplastos, principalmente a proteínas, e são, normalmente, mascarados pela presença de outros pigmentos clorofílicos dominantes (Silva et al., 2010).

$\mathrm{O}$ índice de polifenóis totais diferiu estatisticamente entre todas as amostras. Observou-se que à medida que se aumentou a proporção de polpa de acerola nos blends $(116,27,201,64$ e 240,50 GAEg/100 g), aumentaram também os valores de polifenóis. Teores de polifenóis totais em polpa de acerola foram estudados por Vieira et al. (2011), que observaram valores de 835,25 e 449,63 GAEg/100 g, para extração aquosa e hidroalcoólica, respectivamente. Almeida et al. (2018) analisaram duas variedades de polpa de jabuticaba e encontraram valores de 1.480,67 (Myrciaria jabuticaba) e 249,82 GAEg/100 g (Myrciaria grandifolia) para polifenóis totais. Certas condições podem interferir nas concentrações de compostos fenólicos, como fatores genéticos, condições ambientais e grau de maturidade dos frutos, bem como o processamento e o armazenamento (Sánchez-Moreno, 2002), podendo ser esse o fator das diferenças verificadas.

\subsection{Caracterização física e físico-química das geleias}

$\mathrm{O}$ teor de água encontrado nas geleias indicou que a amostra $\mathrm{F}_{4}$ apresentou o menor valor, 21,41\% ( Tabela 2), pois a polpa da jabuticaba foi adicionada da casca, conferindo maior quantidade de sólidos totais em relação às demais formulações, com variação entre $26,77 \%$ a $29,94 \%$. Quatro formulações de geleias de jabuticaba (polpa/casca) foram caracterizadas por Prasniewski et al. (2017), que verificarm um teor de água em torno de $20 \%$, valor equiparado à geleia $\mathrm{F}_{4}(21,41 \%)$, que contém apenas jabuticaba.

Os valores de cinzas das geleias variaram de $0,63 \%$ a $0,76 \%$, sendo o maior valor encontrado para a geleia elaborada apenas com polpa de acerola. Dessimoni-Pinto et al. (2011), ao elaborarem geleias a partir de polpa de jabuticaba, identificaram $0,09 \%$ de cinzas, enquanto a geleia constituída de $100 \%$ jabuticaba com adição de cascas apresentou $0,66 \%$, valor bem superior. O teor de cinzas depende do tipo de solo em que a frutífera for cultivada e da composição do mesmo (Khan et al., 2014). 
A quantidade adicionada de inulina não interferiu nas características físico-químicas, assim como na geleia de maçã e geleia de abacaxi (Santos et al., 2014), quanto aos teores de umidade e cinzas (Lachman et al., 2014).

Os valores de atividade de água foram superiores a 0,77 , o que evidencia a necessidade da adição de conservante para garantir a segurança do produto contra desenvolvimento microbiano. Desta forma, faz-se necessária a adição do benzoato de potássio nas formulações das geleias prebióticas tradicionais mistas de jabuticaba e acerola.

Tabela 2. Caracterização físico-química das geleias prebióticas tradicionais mistas de jabuticaba e acerola.

\begin{tabular}{|c|c|c|c|c|c|c|c|}
\hline \multirow{2}{*}{ Parâmetros } & \multicolumn{7}{|c|}{ Formulações das geleias tradicionais } \\
\hline & $\mathrm{F}_{1}$ & $\mathrm{~F}_{2}$ & $\mathrm{~F}_{3}$ & $\mathrm{~F}_{4}$ & $\mathrm{~F}_{5}$ & $\mathrm{CV} \%$ & MG \\
\hline Teor de água (\%) & $29,68^{\mathrm{a}}$ & $29,94^{\mathrm{a}}$ & $26,77^{\mathrm{b}}$ & $21,41^{\mathrm{c}}$ & $29,84^{\mathrm{a}}$ & 2,76 & 27,52 \\
\hline Cinzas $(\%)$ & $0,66^{\mathrm{bc}}$ & $0,63^{\mathrm{c}}$ & $0,72^{\mathrm{ab}}$ & $0,66^{\mathrm{bc}}$ & $0,76^{\mathrm{a}}$ & 5,04 & 0,68 \\
\hline Aw & $0,785^{\mathrm{b}}$ & $0,774^{\mathrm{c}}$ & $0,738^{\mathrm{d}}$ & $0,713^{\mathrm{e}}$ & $0,795^{\mathrm{a}}$ & 0,21 & 0,76 \\
\hline $\operatorname{ATT}(\%)$ & $0,77^{\mathrm{a}}$ & $0,80^{\mathrm{a}}$ & $0,77^{\mathrm{a}}$ & $0,78^{\mathrm{a}}$ & $0,80^{\mathrm{a}}$ & 3,38 & 0,78 \\
\hline $\mathrm{pH}$ & $3,03^{\mathrm{a}}$ & $2,87^{\mathrm{a}}$ & $3,27^{\mathrm{a}}$ & $3,17^{\mathrm{a}}$ & $3,01^{\mathrm{a}}$ & 8,52 & 3,06 \\
\hline SST $\left({ }^{\circ}\right.$ Brix $)$ & $65,00^{c}$ & $66,00^{\mathrm{b}}$ & $68,00^{\mathrm{a}}$ & $68,00^{\mathrm{a}}$ & $65,00^{\mathrm{c}}$ & 0,19 & 66,37 \\
\hline AR (\%) & $30,57^{\mathrm{e}}$ & $35,42^{\mathrm{d}}$ & $41,03^{\mathrm{c}}$ & $55,30^{\mathrm{a}}$ & $46,52^{b}$ & 1,27 & 41,76 \\
\hline AT $(\%)$ & $50,02^{\mathrm{d}}$ & $55,93^{\mathrm{c}}$ & $61,13^{\mathrm{a}}$ & $59,52^{\mathrm{ab}}$ & $57,92^{\mathrm{bc}}$ & 0,03 & 54,3 \\
\hline Vitamina C (mg/100 g) & $380,53^{b}$ & $367,87^{\mathrm{c}}$ & $332,16^{\mathrm{e}}$ & $354,20^{\mathrm{d}}$ & $314,16^{\mathrm{a}}$ & 0,07 & 369,78 \\
\hline $\mathrm{L}^{*}$ & $20,53^{\mathrm{a}}$ & $18,72^{\mathrm{cd}}$ & $19,22^{\mathrm{bc}}$ & $17,93^{\mathrm{d}}$ & $19,95^{\mathrm{ab}}$ & 1,73 & 19,27 \\
\hline$+a^{*}$ & $4,58^{\mathrm{a}}$ & $3,16^{\mathrm{b}}$ & $2,92^{\mathrm{b}}$ & $1,97^{\mathrm{c}}$ & $2,91^{\mathrm{b}}$ & 3,29 & 3,10 \\
\hline$+b^{*}$ & $5,74^{\mathrm{a}}$ & $4,70^{\mathrm{b}}$ & $4,55^{\mathrm{b}}$ & $2,58^{\mathrm{c}}$ & $4,43^{\mathrm{b}}$ & 5,23 & 4,39 \\
\hline Antocianina $(\mathrm{mg} / 100 \mathrm{~g})$ & $1,84^{\mathrm{b}}$ & $1,56^{\mathrm{c}}$ & $1,96^{\mathrm{a}}$ & $1,48^{\mathrm{e}}$ & $1,52^{\mathrm{d}}$ & 0,35 & 1,66 \\
\hline $\mathrm{CT}(\mu \mathrm{g} / \mathrm{g})$ & $2,81^{\mathrm{b}}$ & $3,38^{\mathrm{d}}$ & $1,63^{\mathrm{e}}$ & $2,68^{\mathrm{c}}$ & $4,62^{\mathrm{a}}$ & 0,19 & 3,04 \\
\hline PT (GAEg/100 g) & $329,18^{b}$ & $262,41^{\mathrm{c}}$ & $185,47^{\mathrm{d}}$ & $135,50^{\mathrm{e}}$ & $405,29^{a}$ & 0,13 & 263,57 \\
\hline
\end{tabular}

$\mathrm{F}_{1}=75 \%$ acerola $/ 25 \%$ jabuticaba. $\mathrm{F}_{2}=50 \%$ acerola $/ 50 \%$ jabuticaba. $\mathrm{F}_{3}=25 \%$ acerola $/ 75 \%$ jabuticaba. $\mathrm{F}_{4}=100 \%$ jabuticaba. $\mathrm{F}_{5}=100 \%$ acerola. $\mathrm{A}_{\mathrm{w}}=$ atividade de água. $\mathrm{ATT}=$ acidez total titulável. $\mathrm{SST}=$ sólidos solúveis totais. $\mathrm{AR}=$ açúcar redutor. AT = açúcar total. $+\mathrm{a}^{*}=$ intensidade de vermelho. $+\mathrm{b}^{*}=$ intensidade de amarelo. $\mathrm{L}^{*}=$ luminosidade. $\mathrm{CT}=$ carotenoides totais. $\mathrm{PT}=$ polifenóis totais. $\mathrm{CV}=$ coeficiente de variação. $\mathrm{MG}$ = média geral. Obs.: Médias seguidas da mesma letra na linha não diferem estaticamente pelo teste de Tukey, a $5 \%$ de probabilidade.

Os teores de acidez e pH não diferiram estatisticamente entre todas as amostras. Paiva et al. (2015), ao avaliarem a qualidade de geleia de acerola e melão, observaram $\mathrm{pH}$ em torno de 3,3, o qual se equipara ao $\mathrm{pH}$ das geleias do presente estudo. As geleias a partir de polpas e blends de acerola e jabuticaba indicaram teores de acidez com variação de $0,77 \%$ a $0,80 \%$. Oliveira et al. (2016) formularam geleias de laranja com e sem hortelã, e encontraram valores de $0,51 \%$ e $0,50 \%$, respectivamente. Desta forma, as geleias prebióticas tradicionais mistas de jabuticaba e acerola obtiveram $\mathrm{pH}$ e teor de acidez ideais para formação do gel, pois não foi observado rompimento e nem dificuldade de formação do gel.

A variação de ${ }^{\circ}$ Brix de 65 a 68 nas geleias em questão, a concentração de $1 \%$ de pectina e o pH em torno de 3,0 garantem a formação ótima de gel e a rigidez da estrutura de acordo com o Diagrama de Rauch (Silva, 2017).

O teor de sólidos solúveis das geleias mistas apresentou-se dentro dos padrões admissíveis da legislação brasileira, que estabelece valores mínimos de $65^{\circ} \mathrm{Brix}$, para geleia de frutos. As geleias $\mathrm{F}_{1}$ e $\mathrm{F}_{5}$, as quais possuem a maior concentração de polpa de acerola na formulação, apresentaram SST de $65{ }^{\circ}$ Brix. Comportamento semelhante foi observado por Teles et al. (2017), ao estudarem geleia mista de graviola com adição de pimenta dedo-de-moça, com $0,5 \% ; 1,0 \%$ e $1,5 \%$ de pectina.

Os teores de açúcares redutores (30,57\% a 55,30\%) foram maiores e de açúcares totais $(50,02 \%$ a 59,52\%) foram menores em relação aos valores das geleias prebióticas de maracujá com cenoura encontrados por Gomes et al. (2013) com $16,56 \%$ e $60,00 \%$, respectivamente, exceto para a geleia $F_{3}(61,13 \%)$, elaborada com $25 \%$ acerola $/ 75 \%$ jabuticaba. $\mathrm{O}$ açúcar não redutor utilizado nas formulações (sacarose) sofreu inversão 
parcial, dando origem a açúcares redutores (glicose e frutose), contribuindo para a formação de cor do produto, tanto pela caramelização do açúcar como pela reação de Maillard (proteínas e açúcares redutores) (Vieira et al., 2017).

Quanto aos valores de vitamina $\mathrm{C}$, verificou-se que à medida que se elevou o percentual de polpa de acerola nas geleias, aumentou o valor de vitamina $C$, com oscilações de 332,16 a 380,53 mg/100 g. Contudo, verificou-se que o teor de vitamina $\mathrm{C}$ na geleia de acerola, $314,16 \mathrm{mg} / 100 \mathrm{~g}$, foi menor que na geleia de jabuticaba, $354,20 \mathrm{mg} / 100 \mathrm{~g}$. O consumo das geleias pode complementar a ingestão diária de vitamina C, a qual corresponde a $1.000 \mathrm{mg}$ por dia (Universidade Estadual de Campinas, 2011). Comportamento semelhante foi verificado por Maciel et al. (2009), quanto à proporcionalidade de polpa de acerola na composição de geleias simples e mistas de manga e acerola, para as formulações $50 \%$ manga $/ 50 \%$ acerola, $60 \%$ manga $/ 40 \%$ acerola e $75 \%$ manga $/ 25 \%$ acerola. A distinção de valores entre as geleias acontece, porque a vitamina $\mathrm{C}$ é muito instável, podendo, desta forma, ocorrer alta degradação durante a produção da geleia, visto que são necessárias etapas, como fragmentação, homogeneização e concentração, em elevada temperatura até níveis altos de sólidos solúveis ou completo tratamento térmico de pasteurização (Baker et al., 2005), além dos fatores: pH, oxigênio, luz e teor de umidade ou atividade de água (ElezMartínez \& Martín-Belloso, 2007).

As geleias $F_{1}, F_{2}, F_{3}$ e $F_{5}$, as quais possuem polpa de acerola em suas formulações, apresentaram valores de $L^{*}$ entre 18,72 e 20,53, evidenciando uma polpa mais clara, uma vez que a adição da sacarose aumentou o brilho do produto, em relação à geleia de $100 \%$ jabuticaba $(17,93)$, a qual apresentou tendência a uma cor mais escura, devido à pigmentação arroxeada das cascas do fruto adicionadas e à caramelização do açúcar, com subsequente formação de hidroximetilfurfural, que é um produto intermediário, capaz de sofrer polimerização e capaz de produzir melanina, composto responsável pelo escurecimento do produto (Mamede et al., 2013). Oliveira et al. (2014b) verificaram valores semelhantes para geleias de umbu-cajá, com variações de $L^{*}$ de 14,47 a 23,75. A cor das geleias expressa pelos parâmetros de colorimetria evidencia o índice de transformação natural dos alimentos frescos, assim como as mudanças ocorridas no processo industrial, devido ao escurecimento não enzimático, à reação de Maillard e aos processos de caramelização e destruição de pigmentos (Nogueira \& Jesus, 2014).

Nas formulações das geleias, verificou-se variação de 1,48 a $1,96 \mathrm{mg} / 100 \mathrm{~g}$ e de 1,63 a 4,62 mg/100 g para antocianinas e carotenoides totais, respectivamente (Tabela 2). Os valores representados nas geleias prebióticas tradicionais mistas de jabuticaba e acerola foram menores do que os encontrados nas polpas e blends; desta forma, salienta-se que, durante o processamento de geleias, há liberação de ácidos e enzimas intracelulares que podem entrar em contato com o complexo pigmento-proteína, antecipando a degradação da cor (Cano \& Marin, 1992).

Teores de antocianinas totais e carotenoides totais foram avaliados em geleia convencional de araçávermelho por Reissig et al. (2016), os quais identificaram valores de $2,47 \mathrm{mg} / 100 \mathrm{~g}$ e $26,00 \mu \mathrm{g} / 100 \mathrm{~g}$ de $\beta$-caroteno, respectivamente. Rutz et al. (2012) elaboraram geleia tradicional de fisalis e encontraram teor de 3,94 $\mu \mathrm{g} / 100 \mathrm{~g}$ de carotenoides totais. De acordo com Palioto et al. (2015), pesquisas abordam a relação entre o aumento no consumo de alimentos ricos em carotenoides e antocianinas, e a diminuição no risco de várias doenças, pois os carotenoides são os compostos mais estudados como agentes quimiopreventivos.

Observou-se que, na proporção em que se aumentou a porcentagem de polpa de jabuticaba, o teor de polifenóis totais (PT) diminuiu, visto que a geleia de jabuticaba possui $135,75 \mathrm{GAEg} / 100 \mathrm{~g}$ e a geleia de acerola, 405,43 GAEg/100 g. Falcão et al. (2007), ao desenvolverem geleia de uva com adição de frutose e aspartame, verificaram valores de PT entre 191,5 e 235,4 GAEg/100 g. Ressalta-se que a concentração dos polifenóis nas geleias prebióticas tradicionais mistas de jabuticaba e acerola teve comportamento análogo à polpa de jamelão pasteurizada (Branco et al., 2016). É importante destacar que as polpas de frutas 
compreendem outros componentes antioxidantes, como vitaminas, que podem atuar sinergicamente com fenólicos (Lugasi \& Hóvári, 2003).

A disponibilidade média de polifenóis ofertada para o Brasil é de $48,3 \mathrm{mg} / \mathrm{dia}$, por meio do consumo de chás, cafés e frutas (Faller \& Fialho, 2009); logo, a inserção das geleias mistas na dieta do brasileiro poderia elevar o consumo diário de compostos fenólicos.

Quanto aos parâmetros de textura nas geleias prebióticas tradicionais mistas de jabuticaba e acerola (Tabela 3), a firmeza variou de 0,95 a 4,60 N. De acordo com Gava (2008), a presença do açúcar aumenta a pressão osmótica do meio e, consequentemente, diminui a atividade de água do alimento, bem como remove a camada de água que protege as moléculas de pectina, possibilitando a formação do gel pectina-açúcar. Deve-se averiguar também a quantidade de pectina nas polpas, variação entre $0,73 \%$ a $1,20 \%$ para acerola (França \& Narain, 2003) e 6,18\% para jabuticaba (Vieites et al., 2011). Verificou-se resultado análogo em seis tratamentos de geleia de umbu-cajá elaborada por Oliveira et al. (2014a) a partir de concentrações de açúcar e pectina distintas, com destaque para duas amostras com valores de 3,28 e 3,13 N. Rababah et al. (2012) e Rababah et al. (2014) verificaram a firmeza em geleia de uva e cereja, e encontraram valores de 0,89 e $1,76 \mathrm{~N}$, respectivamente; o parâmetro para geleia de uva está abaixo das amostras do presente trabalho, possivelmente devido à constituição química distinta dos frutos. Entretanto, a firmeza da geleia de cereja corrobora com a geleia $\mathrm{F}_{2}(50 \%$ acerola $/ 50 \%$ jabuticaba) com $1,79 \mathrm{~N}$.

Tabela 3. Perfil de textura das geleias prebióticas mistas de jabuticaba e acerola.

\begin{tabular}{ccccccc}
\hline \multirow{2}{*}{ Amostras } & \multicolumn{5}{c}{ Perfil de textura } \\
\cline { 2 - 7 } & FIR $(\mathrm{N})$ & ADE $(\mathrm{N})$ & COE $(\mathrm{N})$ & ELA $(\mathrm{N})$ & GOM $(\mathrm{N})$ & MAS (N) \\
\hline $\mathrm{F}_{1}$ & $1,24^{\mathrm{c}}$ & $0,93^{\mathrm{c}}$ & $0,38^{\mathrm{bc}}$ & $1,00^{\mathrm{a}}$ & $0,48^{\mathrm{d}}$ & $0,48^{\mathrm{d}}$ \\
$\mathrm{F}_{2}$ & $1,79^{\mathrm{bc}}$ & $1,45^{\mathrm{b}}$ & $0,45^{\mathrm{ab}}$ & $0,98^{\mathrm{a}}$ & $0,84^{\mathrm{c}}$ & $0,84^{\mathrm{c}}$ \\
$\mathrm{F}_{3}$ & $2,49^{\mathrm{b}}$ & $1,99^{\mathrm{a}}$ & $0,52^{\mathrm{a}}$ & $0,99^{\mathrm{a}}$ & $1,32^{\mathrm{b}}$ & $1,42^{\mathrm{b}}$ \\
$\mathrm{F}_{4}$ & $4,60^{\mathrm{a}}$ & $2,34^{\mathrm{a}}$ & $0,41^{\mathrm{bc}}$ & $1,00^{\mathrm{a}}$ & $1,97^{\mathrm{a}}$ & $2,04^{\mathrm{a}}$ \\
$\mathrm{F}_{5}$ & $0,95^{\mathrm{c}}$ & $0,60^{\mathrm{c}}$ & $0,34^{\mathrm{c}}$ & $1,00^{\mathrm{a}}$ & $0,33^{\mathrm{d}}$ & $0,33^{\mathrm{e}}$ \\
$\mathrm{MG}_{\mathrm{CV} \%}$ & 2,21 & 1,46 & 0,42 & 0,99 & 0,99 & 1,02 \\
\hline
\end{tabular}

FIR $=$ Firmeza $. \mathrm{ADE}=$ Adesividade. $\mathrm{COE}=$ Coesividade. $\mathrm{ELA}=$ Elasticidade $. \mathrm{GOM}=$ Gomosidade $. \mathrm{MAS}=$ Mastigabilidade. $\mathrm{MG}=$ Média geral. CV $(\%)=$ Coeficiente de variação. Obs.: Médias seguidas das mesmas letras nas linhas não diferem estaticamente pelo teste de Tukey, a $5 \%$ de probabilidade.

As amostras $F_{1}$ e $F_{5}$, e $F_{3}$ e $F_{4}$ não diferiram estatisticamente entre si quanto à adesividade, uma vez que essas formulações constam das maiores quantidades de acerola e jabuticaba, respectivamente, evidenciando o comportamento semelhante.

Garrido et al. (2015) elaboraram geleias de maçã com tratamentos distintos quanto à concentração de pectina e observaram valores de 0,37 a $0,53 \mathrm{~N}$ para coesividade, 0,89 a $0,96 \mathrm{~N}$ para elasticidade e $0,30 \mathrm{a} 1,90 \mathrm{~N}$ para gomosidade. Comportamento semelhante foi verificado quanto a coesividade $\mathrm{e}$ gomosidade para as geleias prebióticas mistas de jabuticaba e acerola.

À medida que se aumenta o valor da mastigabilidade, aumenta-se a energia requerida para mastigar a geleia. Desta forma, $\mathrm{F}_{3}(1,42 \mathrm{~N})$ e $\mathrm{F}_{4}(2,04 \mathrm{~N})$, maiores porcentagens de polpa de jabuticaba, apresentaram tal comportamento devido à consistência mais firme da jabuticaba. Curi et al. (2017), ao estudarem geleias elaboradas a partir de cinco variedades de pera, obtiveram valores menores quanto à mastigabilidade com variações de 0,03 a $0,45 \mathrm{~N}$. Há algumas razões que explicam a variação de textura entre geleias, tais como açúcar, $\mathrm{pH}$, acidez e conteúdo de pectina solúvel, que são os principais fatores que podem influenciar a gelificação e a textura do produto (Souza et al., 2014).

A geleia $\mathrm{F}_{4}$, obtida a partir de polpa e casca de jabuticaba, apresentou maiores valores para as propriedades de textura, exceto para coesividade, pois a polpa da jabuticaba é mucilaginosa e adocicada, e juntamente com a adição da sacarose, foi capaz de formar um gel mais denso e firme. A concentração de sólidos solúveis nas 
geleias afeta sua textura, tanto pela redução do teor de água, com aumento da rigidez da estrutura (Glicksman, 1969), quanto pela necessidade de evaporação de uma quantidade maior da água da geleia, devido ao tempo de cocção e à temperatura do produto final. Desta maneira, aumentam as chances de a pectina ser hidrolisada pela concentração de ácido (Jackix, 1988), o que torna a estrutura descontínua e mais aderente (Alikonis, 1979; Jackix, 1988).

\section{Conclusão}

As polpas de jabuticaba e acerola podem ser utilizadas com na produção de geleias tradicionais, devido ao bom desempenho das suas propriedades no perfil de textura, além da coloração que garante um atrativo ao consumidor e da presença de pigmentos que combatem os radicais livres.

Todas as formulações apresentadas são uma excelente forma de conservar a fruta, por apresentar elevada acidez, baixo $\mathrm{pH}$ e reduzido teor de água, o que garante uma geleia de qualidade com as características físicasquímicas de acordo com a legislação, com viabilidade para produção em nível industrial.

As geleias prebióticas tradicionais mistas de jabuticaba e acerola apresentam potencial antioxidante, pois apresentaram teores relevantes de vitamina $\mathrm{C}$ e antocianinas. Ademais, todas as geleias, mistas e simples, apresentaram teores consideráveis de carotenoides e polifenóis totais.

Quanto ao perfil funcional, o produto atende à legislação brasileira em vigor por conter inulina, além da presença de antioxidantes. Desta forma, a utilização da polpa de blends de jabuticaba e acerola na fabricação de geleias é uma alternativa tecnológica e nutricionalmente viável.

\section{Referências}

Alikonis, J. J. (1979). Starch and pectin jellies. In J. J. Alikonis, Candy technology (Cap. 10, pp. 109-118). Connecticut: AVI. Almeida, E. S., Silva, R. J. N., \& Gonçalves, E. M. (2018). Compostos fenólicos totais e características físico-químicas de frutos de jabuticaba. Gaia Scientia, 12(1), 81-89. http://dx.doi.org/10.22478/ufpb.1981-1268.2018v12n1.30418

Aquino, A. C. M. S., Móes, R. S., \& Castro, A. A. (2011). Estabilidade de ácido ascórbico, carotenoides e antocianinas de frutos de acerola congelados por métodos criogênicos. Brazilian Journal of Food Technology, 14(2), 154-163. http://dx.doi.org/10.4260/BJFT2011140200020

Baker, R. A., Berry, N., Hui, Y. H., \& Barret, D. M. (2005). Fruit preserves and jams in processing fruit: Science and technology (2. ed.). New York: CRC Press.

Barbosa, P. S., Andrade, E. S., Jesus, J. H., Brondani, F. M. M., \& Vieira, R. (2017). Análise e quantificação do teor alcoólico do fermentado artesanal de jabuticaba. Revista Científica da Faculdade de Educação e Meio Ambiente, 8(1), 16-32. http://dx.doi.org/10.31072/rcf.v8i1.458

Becker, F. S., Vilas Boas, A. C., Sales, A., Tavares, L. S., Siqueira, H. H., \& Vilas Boas, E. V. B. (2015). Caracterização de Jabuticaba 'Sabará' em diferentes estádios de maturação. Acta Scientiarum. Agronomy, 37(4), 457-462. http://dx.doi.org/10.4025/actasciagron.v37i4.19499

Branco, I. G., Moraes, I. C. F., Argandoña, E. J. S., Madrona, G. S., Santos, C., Ruiz, A. L. T. G., Carvalho, J. E., \& Haminiuk, C. W. I. (2016). Influence of pasteurization on antioxidant and in vitro anti-proliferative effects of jambolan (Syzygium cumini (L.) Skeels) fruit pulp. Industrial Crops and Products, 89(2), 225-230. http://dx.doi.org/10.1016/j.indcrop.2016.04.055

Brasil. Ministério da Saúde. Agência Nacional de Vigilância Sanitária - ANVISA. (2005, setembro 23). Resolução de Diretoria Colegiada $n^{\circ} 272$, de 22 de setembro de 2005. Regulamento técnico para produtos de vegetais, produtos de frutas e cogumelos comestíveis. Diário Oficial [da] República Federativa do Brasil, Brasília. Seção 1, pt I.

Caetano, P. K., Daiuto, E. R., \& Vieites, R. L. (2012). Característica físico-química e sensorial de geleia elaborada com polpa e suco de acerola. Brazilian Journal of Food Technology, 15(3), 191-197. http://dx.doi.org/10.1590/S1981-67232012005000011

Cano, M. P., \& Marin, M. A. (1992). Pigment composition and colour of frozen and canned kiwi fruit slices. Journal of Agricultural and Food Chemistry, 40(11), 2141-2146. http://dx.doi.org/10.1021/jf00023a020

Chaito, C., Judprasong, K., \& Puwastien, P. (2014). Inulin content of fortified food products in Thailand. Food Chemistry, 3(1), 102-105. PMid:26433294.

Chitarra, M. I., \& Chitarra, A. B. (2005). Pós-colheita de frutos e hortaliças: Fisiologia e manuseio (2. ed.). Lavras: UFLA.

Curi, P. N., Bisi, R. B., Salgado, D. L., Barbosa, C. M. A., Pio, R., \& Souza, V. R. (2017). Hybrid cultivars of pear in subtropics regions: Processing ability in the form of jelly. Ciência Rural, 47(11), 1-7. http://dx.doi.org/10.1590/0103-8478cr20170331

Dessimoni-Pinto, N. A. V., Moreira, W. A., Cardoso, L. M., \& Pantoja, L. A. (2011). Jaboticaba peel for jelly preparation: An alternative technology. Food Science and Technology, 31(4), 864-869. http://dx.doi.org/10.1590/S0101-20612011000400006 
Dias, C. S., Borges, S. V., Queiroz, F., \& Pereira, P. A. P. (2011). Influência da temperatura sobre as alterações físicas, físicoquímicas e químicas de geleia da casca de banana (Musa spp.) Cv. Prata durante o armazenamento. Revista do Instituto Adolfo Lutz, 70(1), 28-34.

Elez-Martínez, P., \& Martín-Belloso, O. (2007). Effects of high intensity pulsed electric field processing conditions on vitamin C and antioxidant capacity of orange juice and gazpacho, a cold vegetable soup. Food Chemistry, 102(1), 201-209.

http://dx.doi.org/10.1016/j.foodchem.2006.04.048

Falcão, A. P., Chaves, E. S., Kuskoski, E. M., Fett, R., Falcão, L. D., \& Bordignon-Luiz, M. T. (2007). Índice de polifenóis, antocianinas totais e atividade antioxidante de um sistema modelo de geleia de uvas. Food Science and Technology, 27(3), 637-642. http://dx.doi.org/10.1590/S0101-20612007000300032

Faller, A. L. K., \& Fialho, E. (2009). Disponibilidade de polifenóis em frutas e hortaliças consumidas no Brasil. Revista de Saude Publica, 43(2), 211-218. PMid:19225692. http://dx.doi.org/10.1590/S0034-89102009005000010

Ferreira, R. M. A., Aroucha, E. M. M., Góis, V. A., Silva, D. K., \& Sousa, C. M. G. (2011). Qualidade sensorial de geleia mista de melancia e tamarindo. Revista Caatinga, 24(2), 202-206.

Figueiredo Neto, A., Irmão, M. A. S., Alencar Junior, J. P., \& Carneiro, R. M. (2017). Resistance evaluation of the acerola (Malphigia emarginata D.C.) seed to compression. The Journal of Agricultural Science, 9(8), 167-173. http://dx.doi.org/10.5539/jas.v9n8p167

França, V. C., \& Narain, N. (2003). Caracterização química dos frutos de três matrizes de acerola (Malpighia emarginata D.C.). Food Science and Technology, 23(2), 157-160. http://dx.doi.org/10.1590/S0101-20612003000200009

Francis, F. J. (1982). Analysis of anthocyanins. In P. Markakis (Ed.), Anthocyanins as food colors (pp. 181-207). New York: Academic Press. http://dx.doi.org/10.1016/B978-0-12-472550-8.50011-1.

Furlaneto, K. A., Ramos, J. A., Daiuto, E. R., Vieites, R. L., \& Carvalho, L. R. (2015). Elaboração e aceitabilidade da geleia convencional e light de maná cubiu. Nativa: Pesquisas Agrárias e Ambientais, 3(4), 276-280. http://dx.doi.org/10.14583/23187670.v03n04a09

Garrido, J. I., Lozano, J. E., \& Genovese, D. B. (2015). Effect of formulation variables on rheology, texture, colour, and acceptability of apple jelly: Modelling and optimization. Food Science and Technology, 62(1), 325-332.

Gava, A. J. (2008). Tecnologia de alimentos: Princípios e aplicações. São Paulo: Nobel.

Glicksman, M. (1969). Gum technology in the food Industry (Cap. 6. Pectins, pp. 159-189). New York: Academic Press.

Gomes, R. B., Santos, M. B., Cardoso, R. L., Tavares, J. T. Q., \& Cunha, D. S. (2013). Elaboração e avaliação físico-químico e sensorial de geleia de maracujá com cenoura. Enciclopédia Biosfera, 9(16), 2013-2766.

Instituto Adolfo Lutz - IAL. (2008). Métodos físico-químicos para análise de alimentos (4. ed.). São Paulo: IAL.

Jackix, M. H. (1988). Doces, geleias e frutas em calda. Campinas: Ícone.

Khan, A. A., Sajid, M., \& Rab, A. (2014). Tomato fruit quality as affected by different sources of phosphorus. Pakistan Journal of Nutrition, 13(12), 692-699. http://dx.doi.org/10.3923/pjn.2014.692.699

Lachman, C., Galvão, R., Cristo, T. W., Brecailo, M. K., Santos, E. F., Silva, E. C., Manhani, M. R., \& Novello, D. (2014). Geleia de maçã adicionada de inulina: Parâmetros físico-químicos e avaliação sensorial entre crianças. Revista da Universidade Vale do Rio Verde, 12(1), 57-69. http://dx.doi.org/10.5892/ruvrd.v12i1.1225

Lamounier, M. L., Andrade, F. C., Mendonça, C. D., \& Magalhães, M. L. (2015). Desenvolvimento e caracterização de diferentes formulações de sorvetes enriquecidos com farinha da casca da jabuticaba (Myrciaria cauliflora). Revista do Instituto de Latícinios Cândido Tostes, 70(2), 93-104. http://dx.doi.org/10.14295/2238-6416.v70i2.400

Lima, A. J. B., Corrêa, A. D., Alves, A. P. C., Abreu, C. M. P., \& Dantas-Barros, A. M. (2008). Caracterização do fruto jabuticaba (Myrciaria cauliflora) e de suas frações. Archivos Latinoamericanos de Nutricion, 58(4), 416-421.

Lima, A. J. B., Corrêa, A. D., Dantas-Barros, A. M., Nelson, D. L., \& Amorim, A. C. L. (2011). Sugars, organic acids, minerals and lipids in jabuticaba. Revista Brasileira de Fruticultura, 33(8), 540-550. http://dx.doi.org/10.1590/S0100-29452011000200026

Lugasi, A., \& Hóvári, J. (2003). Antioxidant properties of commercial alcoholic and nonalcoholic beverages. Die Nahrung, 47(2), 79-86. PMid:12744283. http://dx.doi.org/10.1002/food.200390031

Maciel, M. I. S., Melo, E. A., Lima, V. L. A. G., Silva, W. S., Maranhão, C. M. C., \& Souza, K. A. (2009). Características sensoriais e físico-químicas de geleias mistas de manga e acerola. Boletim do Centro de Pesquisa e Processamento de Alimentos, 27(2), 247-256. http://dx.doi.org/10.5380/cep.v27i2.22035

Malegori, C., Marques, E. J. N., Freitas, S. T., Pimentel, M. F., Pasquini, C., \& Casiraghi, E. (2017). Comparing the analytical performances of Micro-NIR and FT-NIR spectrometers in the evaluation of acerola fruit quality, using PLS and SVM regression algorithms. Talanta, 165, 112-116. PMid:28153229. http://dx.doi.org/10.1016/j.talanta.2016.12.035

Mamede, M. E. O., Dib De Carvalho, L., Viana, E. S., Alves, L. O., Soares, W. S. F., \& Ritzinger, R. (2013). Production of dietetic jam of umbu-caja (Spondias $s p$.): Physical, physicochemical and sensorial evaluations. Food and Nutrition Sciences, 4(4), 461-468. http://dx.doi.org/10.4236/fns.2013.44059

Mercali, G. D., Sarkis, J. R., Jaeschke, D. P., Tessaro, I. C., \& Marczak, L. D. F. (2011). Physical properties of acerola and blueberry pulps. Journal of Food Engineering, 106(1), 283-289. http://dx.doi.org/10.1016/j.jfoodeng.2011.05.010

Monteiro, R. C. R., Veloso, C. R., Neres, L. S., Lourenço Júnior, J. B., Pacheco, E. A., Abe Sato, S. T., Santos, M. A. S. Nahum, B. S., \& Ribeiro, I. A. (2015). Desenvolvimento e avaliação da qualidade de sorvete de iogurte simbiótico, de leite de búfala enriquecido com polpa de açaí (Euterpe oleracea). Nucleus, 12(2), 237-244. http://dx.doi.org/10.3738/1982.2278.1162 
Munhoz, C. L., Borges, G. S., Silva, M. L. F., \& Oliveira, R. F. (2018). Avaliação sensorial de iogurtes de jambo vermelho. Revista Inova Ciência \& Tecnologia, 4(1), 25-31.

Nóbrega, E. M., Oliveira, E. L., Genovese, M. I., \& Correia, R. T. P. (2015). The Impact of hot air drying on the physicalchemical characteristics, bioactive compounds and antioxidant activity of acerola (Malphigia emarginata) residue. Journal of Food Processing and Preservation, 39(2), 131-141. http://dx.doi.org/10.1111/jfpp.12213

Nogueira, J. P., \& Jesus, M. A. C. L. (2014). Desenvolvimento, avaliação físico-química, sensorial e colorimétrica da geleia de seriguela diet. Revista Brasileira de Tecnologia Agroindustrial, 8(2), 1531-1544. http://dx.doi.org/10.3895/S1981$36862014000200007 \mathrm{~S} 1$

Nunes, J. S., Castro, D. S., Sousa, F. C., Silva, L. M. M., \& Gouveia, J. P. G. (2014). Obtenção e caracterização físico-química de polpa de jabuticaba (Myrciaria Cauliflora Berg) congelada. Revista Verde de Agroecologia e Desenvolvimento Sustentável, 9(1), 234-237.

Oliveira, E. N. A., Rocha, A. P. T., Gomes, J. P., \& Santos, D. C. (2014a). Influência das variáveis de processo nas características físicas e químicas de geleias de umbu-cajá. Bioscience Journal, 30(6), 1698-1710.

Oliveira, E. N. A., Santos, D. C., Rocha, A. P. T., Gomes, J. P., \& Silva, W. P. (2014b). Estabilidade de geleias convencionais de umbu-cajá durante o armazenamento em condições ambientais. Revista Brasileira de Engenharia Agrícola e Ambiental, 18(3), 329-337. http://dx.doi.org/10.1590/S1415-43662014000300013

Oliveira, F. A. A., Oliveira, E. N. A., Rodrigues, F. F. G., Feitosa, B. F., \& Almeida, F. L. C. (2017). Caraterização físico-química e sensorial de bebidas funcionais mistas de graviola com água de coco. Energia na Agricultura, 32(3), 301-308. http://dx.doi.org/10.17224/EnergAgric.2017v32n3p301-308

Oliveira, M. M. T., Braga, T. R., Pinheiro, G. K., Silva, L. R., Vieira, C. B., \& Torres, L. B. V. (2016). Parâmetros físico-químicos, avaliação microbiológica e sensorial de geleias de laranja orgânica com adição de hortelã. Revista de la Facultad de Agronomía, 115(1), 29-34.

Paiva, C. A., Aroucha, E. M. M., Ferreira, R. M. A., Araújo, N. O., \& Silva, P. S. L. (2015). Alterações físico-químicas de geleias de melão e acerola durante o armazenamento. Revista Verde, 10(3), 18-23. http://dx.doi.org/10.18378/rvads.v10i3.3495

Palioto, G. F., Silva, C. F. G., Mendes, M. P., Almeida, V. V., Rocha, C. L. M. S. C., \& Tonin, L. T. D. (2015). Composição centesimal, compostos bioativos e atividade antioxidante de frutos de Morinda citrifolia Linn (noni) cultivados no Paraná. Revista Brasileira de Plantas Medicinais, 17(1), 59-66. http://dx.doi.org/10.1590/1983-084X/13_066

Pelegrine, D. H. G., Andrade, M. S., \& Nunes, S. H. (2015). Elaboração de geleias a partir de misturas binárias compostas pelas polpas de laranja e acerola. Ciência e Natura, 37(1), 124-129. http://dx.doi.org/10.5902/2179460X15051

Prasniewski, A., Cartabiano, C. E., Pegorini, D., Roncatti, R., \& Pereira, E. A. (2017). Aproveitamento tecnológico da casca de jabuticaba na elaboração de geleia. Synergismus Scyentifica, 12(1), 74-80.

Rababah, T. M., Al-U'Datt, M., Al-Mahasneh, M., Yang, W., Feng, H., Ereifej, K., Kilani, I., \& Ishmais, M. A. (2014). Effect of jam processing and storage on phytochemicals and physiochemical properties of cherry at different temperatures. Journal of Food Processing and Preservation, 38(1), 247-254. http://dx.doi.org/10.1111/j.1745-4549.2012.00770.x

Rababah, T. M., Al-u'datt, M., Almajwal, A., Brewer, S., Feng, H., Al-Mahasneh, M., Ereifej, K., \& Yang, W. (2012). Evaluation of the nutraceutical, physiochemical and sensory properties of raisin jam. Journal of Food Science, 77(6), 609-613.

PMid:22582920. http://dx.doi.org/10.1111/j.1750-3841.2012.02708.x

Ramalho, A. S. T. M. (2005). Sistema funcional de controle de qualidade a ser utilizado como padrão na cadeia de comercialização de laranja Pêra (Citrussinensis L. Osbeck) (Dissertação de mestrado). Universidade de São Paulo, São Paulo. Ranganna, S. (1986). Handbook of analysis and quality control for fruits and vegetables products. New Delhi: Tata Mc Graw Hill Publishing.

Reis, D. S., Figueiredo Neto, A., Costa, J. D. S., Almeida, F. A. C., \& Gouvêia, J. P. G. (2017). Desorption isotherms of acerola fruits variety 'Okinawa'. Cientifica (Jaboticabal), 45(2), 130-136. http://dx.doi.org/10.15361/1984-5529.2017v45n2p130-136

Reissig, G. N., Vergara, L. P., Franzon, R. C., Rodrigues, R. S., \& Chim, J. F. (2016). Bioactive compounds in conventional and no added sugars red strawberry guava (Psidium cattleianum Sabine) jellies. Revista Brasileira de Fruticultura, 38(3), 1-7. http://dx.doi.org/10.1590/0100-29452016062

Rosso, V. V., \& Mercadante, A. Z. (2007). The high ascorbic acid content is the main cause of the low stability of anthocyanin extracts from acerola. Food Chemistry, 103(3), 935-943. http://dx.doi.org/10.1016/j.foodchem.2006.09.047

Rufino, M. S. M., Alves, R. E., Fernandes, F. A. N., \& Brito, E. S. (2011). Free radical scavenging behavior of ten exotic tropical fruits extracts. Food Research International, 44(7), 2072-2075. http://dx.doi.org/10.1016/j.foodres.2010.07.002

Rutz, J. K., Voss, G. B., Jacques, A. C., Pertuzatti, P. B., Barcia, M. T., \& Zambiazi, R. C. (2012). Caracterização de geleia de Physalis peruviana L. Alimentos e Nutrição, 23(3), 369-375.

Salamoni, B. F., Boas, A. C. V., Adones, S., Tavares, L. S., Siqueira, H. H., \& Boas, E. V. B. V. (2015). Caracterização de Jabuticaba 'Sabará' em diferentes estádios de maturação. Acta Scientiarum, 37(4), 457-460.

Sánchez-Moreno, C. (2002). Compuestos polifenólicos: Estructura y clasificación, presencia en alimentos y consumo, biodisponibilidad y metabolismo. Alimentaria: Revista de Tecnología e Higiene de los Alimentos, 2(329), 19-28.

Santos, K. A., Faix, P. N., Santos, E. F., Manhani, M. R., Silva, E. C., \& Novello, D. (2014). Efeito da adição de inulina em geleia de abacaxi: Análise físico-química e sensorial entre escolares. O Mundo da Saude, 38(3), 286-295. http://dx.doi.org/10.15343/0104-7809.20143803286295

Silva, F. A. Z., \& Azevedo, C. A. V. (2016). The assistat software version 7.7 and its use in the analysis of experimental data. African Journal of Agricultural Research, 11(39), 3733-3740. http://dx.doi.org/10.5897/AJAR2016.11522 
Silva, F. S. (2017). Elaboração de geleía com mix de polpa de cagaita (Eugenia dysenterica) e mangaba (Hancornia speciosa) e avaliação dos parâmetros de qualidade (Dissertação de mestrado). Universidade Federal de Tocantins, Palmas.

Silva, M. L. C., Costa, R. S., Santana, A. S., \& Koblitz, M. G. B. (2010). Compostos fenólicos, carotenóides e atividade antioxidante em produtos vegetais. Semina: Ciências Agrárias, 31(3), 669-682. http://dx.doi.org/10.5433/16790359.2010v31n3p669

Singleton, V. L., \& Rossi, J. A. (1965). Colorimetry of total phenolics with phosphomolybdic-phosphotungstic acid reagents. American Journal of Enology and Viticulture, 20(2), 144-158.

Souza, V. R., Pereira, P. A. P., Pinheiro, A. C. M., Lima, L. C. O., Pio, R., \& Queiroz, F. (2014). Analysis of the subtropical blackberry cultivar potential in jelly processing. Journal of Food Science, 79(9), 1776-1781. PMid:25154800. http://dx.doi.org/10.1111/1750-3841.12565

Teles, A. C. M., Pinto, E. G. A., Santos, J. R., Oliveira, C. F. D., \& Soares, D. S. B. (2017). Desenvolvimento e caracterização físico-química de geleia comum e extra de graviola com pimenta. Revista de Agricultura Neotropical, 4(1), 72-77. http://dx.doi.org/10.32404/rean.v4i1.1341

Uchoa, A. M. A., Costa, J. M. C., Maia, G. A., Silva, E. M. C., Carvalho, A. F. F. U., \& Meira, T. R. (2008). Parâmetros físicoquímicos, teor de fibra bruta e alimentar de pós alimentícios obtidos de resíduos de frutas tropicais. Segurança Alimentar e Nutricional, 15(2), 58-65. http://dx.doi.org/10.20396/san.v15i2.1817

Universidade Estadual de Campinas - UNICAMP. (2011). TACO: Tabela Brasileira de Composição dos Alimentos (4. ed. rev. ampl.). Campinas: Núcleo de Estudos e Pesquisas em Alimentação.

Uyeda, M., Buonom, H. C. D., Gonzaga, M. F. N., \& Carvalho, F. L. O. (2017). Probióticos e prebióticos: Benefícios acerca da literatura. Revista de Saúde UniAGES, 1(1), 33-57.

Viana, E. S., Jesus, J. L., Reis, R. C., Andrade, M. V. S., \& Sacramento, C. K. (2014). Physicochemical and sensory characterization of banana and araçá-boi jam. Food and Nutrition Sciences, 5(8), 733-741. http://dx.doi.org/10.4236/fns.2014.58083

Vieira, E. C. S., Silva, E. P., Amorim, C. C. M., Sousa, G. M., Becker, F. S., \& Damiani, C. (2017). Aceitabilidade e características físico-químicas de geleia mista de casca de abacaxi e polpa de pêssego. Científica, 45(2), 115-122. http://dx.doi.org/10.15361/1984-5529.2017v45n2p115-122

Vieira, L. M., Sousa, M. S. B., Mancini-Filho, J., \& Lima, A. (2011). Fenólicos totais e capacidade antioxidante in vitro de polpas de frutos tropicais. Revista Brasileira de Fruticultura, 33(3), 888-897. http://dx.doi.org/10.1590/S0100-29452011005000099

Vieites, R. L., Daiuto, E. R., Moraes, M. R., Neves, L. C., \& Carvalho, L. R. (2011). Caracterização físico-química, bioquímica e funcional da jabuticaba armazenada sob diferentes temperaturas. Revista Brasileira de Fruticultura, 33(2), 362-375. http://dx.doi.org/10.1590/S0100-29452011000200006

Volp, A. C. P., Renhe, I. R. T., Barra, K., \& Stringueta, P. C. (2008). Flavonoides antocianinas: Características e propriedades na nutrição e saúde. Revista Brasileira de Nutrição Clínica, 23, 141-149. 